\title{
A Novel PTS Scheme for PAPR Reduction of Filtered-OFDM Signals without Side Information
}

\author{
Yaqiu PENG, Mingqi LI
}

\begin{abstract}
In this paper, a novel partial transmit sequence (PTS) scheme is proposed for reducing the peak-to-average power ratio (PAPR) of filtered orthogonal frequency division multiplexing (f-OFDM) systems. The PTS method is modified such that no side information (SI) transmission is needed. The data and pilot recovery are accomplished by a simple detector, making use of the correlation property of the Hadamard sequence and the transparency property of the pilot signal and an iterative phase detection is further added in a fading channel. Simulation results show that the modified solution provides a higher correct detection probability without increasing the system complexity nor affecting the PAPR suppression performance.
\end{abstract}

Keywords: filtered-OFDM; PAPR; PTS; SI

\section{INTRODUCTION}

Orthogonal frequency division multiplexing (OFDM) has been recognized as an effective waveform for wireless communications. Due to its simple implementation, high spectrum efficiency and proper anti-multipath fading performance, OFDM has been adopted to 3GPP LTE and IEEE 802.11 standard families. Despite its advantages, OFDM suffers from a number of drawbacks such as high out-of-band emissions, high park-to-average power ratio (PAPR).

In the next generation and future communication systems, not only enhanced mobile broadband (eMBB) but also massive machine-type communications (mMTC), ultra-reliable and low latency communications (URLLC) should be supported. To meet various challenging requirements in the physical layer of the next generation communication system, it is essential to introduce new waveforms with more flexible parameter configuration, higher spectral efficiency, lower out-of-band emission (OOBE), lower PAPR, etc. As a result, many new waveforms have been put forward as candidates of NR (new radio), such as Generalized Frequency Division Multiplexing (GFDM) [1], Universal Filtered MultiCarrier (UFMC) [2], Filtered OFDM (f-OFDM) [3], Filterbank Based Multi-Carrier (FBMC) [4], etc. It is worth noting that $\mathrm{f}-\mathrm{OFDM}$ divides the whole system spectrum into several sub-bands and can set parameters like subband width and sub-carrier interval according to different service requirements and scenarios, which make it more flexible to utilize the spectrum resource.

As a newly proposed waveform, f-OFDM not only inherits the advantages of OFDM employed in $4 \mathrm{G}$ but also exhibits distinct flexibility in spectrum utilization according to various service scenarios, backward and forward compatibility, lower OOBE, etc. However, fOFDM also suffers from high PAPR because of the nature of an integration of multiple sub-bands, which leads to the non-efficient amplification of the power amplifier and causes inter-modulation and out-of-band radiation.

In the past decades, various schemes have been proposed to solve the high PAPR problem, which can be divided into two main categories: Signal distortion schemes such as clipping and companding transform [5].
This kind of method is easy to be implemented with low computational complexity, but signal distortion and out-ofband leakage will be introduced at the same time. The other category is schemes without distortion such as Partial transmit sequence (PTS) [6], Select Mapping (SLM) [7] and precoding $[8,9]$. The main idea of the distortion-free algorithms is to optimize PAPR by combining the phase coefficients of the signals with different phase weights. Different from the signal distortion algorithms, the goal of this kind of methods is to reduce the probability of the large peak power signals other than crop the signals. However, the distortion-free algorithms are of high complexity, and some schemes need to transmit side information (SI). The partial transmit sequence (PTS) is more attractive as its distortion-free feature. The basic idea of PTS is that subcarriers are further split into several sub-blocks and then the minimum combination is found based on phase weighting factor via phase rotation. However, to recover signals exactly at the receiver, the transmitter needs to inform the receiver SI, which is the phase weighting factor that can minimize PAPR. The transmission of SI occupies bandwidth. Moreover, the transmitter should increase the transmitting power of SI to ensure accurate transmission. Improper detection of SI will cause major BER performance degradation. All these are detrimental to system efficiency.

Recently, several improved PTS schemes have attempted to remove the transmission of SI at the cost of high computational complexity [10-14]. In [10], the signal sequence is recovered by a maximum likelihood (ML) detector. In [11], the SI is considered as a part of the channel frequency response. Although the scheme can increase the data rate, the conventional equal-spaced pilot tone arrangement has to be improved. Moreover, in [14], a smaller number of phase offsets is adopted by searching in a smaller space, but an ML detector is still required. However, all the above solutions are at the cost of high computational complexity and are based on OFDM architecture. Only a few studies focus on PAPR reduction for the f- OFDM system, most of which are based on the clipping algorithm which will introduce signal distortion. In [15], a precoding scheme has been proposed for universal filtered-orthogonal frequency division multiplexing (UF-OFDM) and f-OFDM. The precoding 
matrix is multiplied to the transmitted signal and the corresponding inverse transformation is carried out to restore the original signal at the receiver. But it has a limited effect on PAPR reduction and can lead to the OOBE problem.

Against this background, the main concern of the PAPR reduction in the $\mathrm{f}-\mathrm{OFMD}$ system is to reduce PAPR with lower complexity without affecting the spectral efficiency and signal distortion. To the best of our knowledge, the PTS scheme without SI has not been used in the f-OFDM system.

In this contribution, a modified PTS scheme targeting for PAPR reduction of f-OFDM signals is proposed. Specifically, at the transmitter, the pilot and data symbols are rotated simultaneously by the phase optimization procedure where the phase optimization factors are selected from the Hadamard matrix. At the receiver, it makes use of the correlation property of the Hadamard sequence and the transparency property of the pilot signal to detect and recover the original data. Compared to the conventional PTS scheme, the proposed one is able to maintain the PAPR performance while without the transmission of SI, thus to mitigate the system overhead.

The rest of this paper is organized as follows: In Section 2, a brief presentation of Filtered-OFDM is given. In Section 3, the original PTS scheme is explained. The new PTS scheme without SI transmission is proposed for $\mathrm{f}-\mathrm{OFDM}$ in Section 4. Section 5 shows the simulation results and conclusions are drawn in Section 6.

\section{THE PRINCIPLE OF FILTERED-OFDM}

To satisfy various service requirements, the spectrum of the f-OFDM system is divided into several links, i.e. sub-bands that can be configured adaptively with different parameters (e.g. subcarrier spacing, the length of $\mathrm{CP}$ and so on) according to the channel characteristics. The mathematical representation of the f-OFDM signal at the transmitter can be written as:

$\tilde{s}_{n}=s(n) \cdot f(n)$ with

$$
s(n)=\sum_{l=0}^{L-1} s_{l}\left[n-l\left(N+N_{c p}\right)\right]
$$

and

$$
s_{l}(n)=\sum_{m=m^{\prime}}^{m^{\prime}+M-1} d_{l, m} \mathrm{e}^{\frac{j 2 \pi m n}{N}}, N_{c p} \leq n \leq N
$$

where $s(n)$ is the original OFDM signal and $f(n)$ is the conjunction of all spectrum-shaping filters on each subband. (2) and (3) are the mathematical representation of OFDM where $d_{l, m}$ denotes the data symbol on the $m^{\text {th }}$ subcarrier of symbol $l$ and $N_{\mathrm{CP}}$ is the CP length.

The major difference between OFDM and f-OFDM is the use of the appropriately designed sub-band filters to suppress OOBE. Furthermore, dynamic parameter setting of $\mathrm{f}-\mathrm{OFDM}$ can also improve the system performance.

\section{CONVENTIONAL PTS SCHEME}

Let $x=\left[x_{0}, x_{1}, \ldots, x_{N-1}\right]^{\mathrm{T}}$ denotes a signal vector in the multi-carrier system with the length of $N$. Peak-toaverage power ratio (PAPR) is defined as

$$
P A P R=\frac{\max _{0 \leq n \leq N-1}\left|x_{n}\right|^{2}}{E\left[\left|x_{n}\right|^{2}\right]}
$$

where $E[$.$] denotes the expectation. The complementary$ cumulative density function (CCDF) has been introduced to quantify PAPR, i.e. the probability that the PAPR exceeds an assigned threshold $\delta$.

$$
C C D F=P(P A P R>\delta)=1-\left(1-\mathrm{e}^{-\alpha \delta}\right)
$$

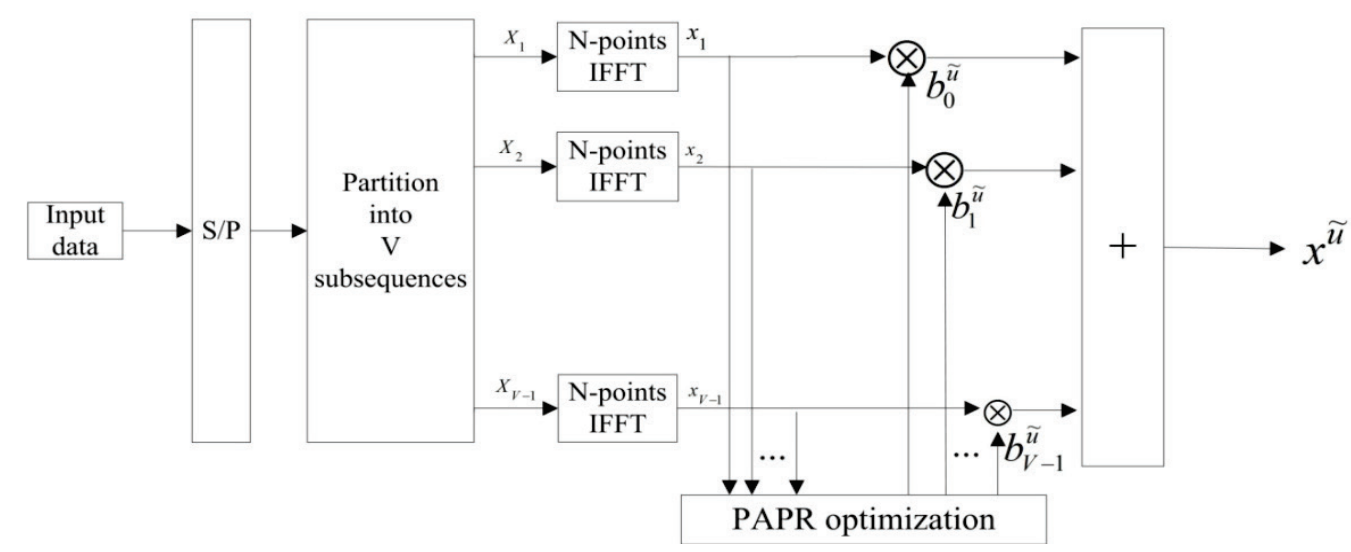

Figure 1 Schematic of conventional PTS scheme

In a multi-carrier system, when the carrier signals are overlapped in the same phase, the instantaneous power of the signal is much higher than the average power, thus leveraging the PAPR significantly. In the PTS scheme, an input symbol with $N$ subcarriers is partitioned into $V$ disjoint subsequences. As shown in Fig. 1, the subcarriers in each subsequence are multiplied by an optimal phase factor chosen from the phase factor set $\boldsymbol{B}$ according to the minimum PAPR criterion. To simplify the actual operation, $\boldsymbol{B}$ is practically $\{ \pm 1\}$ or $\{ \pm 1, \pm j\}$. PTS scheme can reduce 
PAPR without signal distortion, but it shows large computational complexity and needs side information (SI) transmission at the transmitter to ensure correct signal recovery at the receiver, which will increase the redundancy of the system.

\section{PROPOSED PTS SCHEME WITHOUT SI TRANSMISSION}

To remove the transmission of SI with PTS in f-OFDM systems, we resort to the pilot symbols and well-designed phase rotating factors. Due to its good correlation property and low computational complexity, the Hadamard sequence is selected as the phase rotating factor set. Therefore, at the transmitter, the phase optimization with Hadamard sequence is conducted for each sequence, followed by $\mathrm{CP}$ padding and sub-band filtering. At the receiver, benefiting from the correlation property of the Hadamard sequence and the transparency of the pilot signal, the phase factor could be detected and used to recover the data and pilot symbols.

\subsection{Proposed System Model}

In this paper, it is assumed that there are $R$ sub-bands in the f-OFDM system while the numerologies of all the sub-bands are configurable according to scenario requirements in practice. The architecture of the proposed transceiver is illustrated in Fig. 2. $\left\{X_{n}^{r}, n=0,1, \ldots, N_{r}-1, r=1,2, \ldots, R\right\}$ is defined as the $n$ th symbol in the $r$-th sub-band. After the serial-to-parallel conversion, $X_{n}^{r}$ is partitioned into $V$ disjoint subsequences $\quad \boldsymbol{X}_{v}^{r}=\left[X_{v, 0}^{r}, X_{v, 1}^{r}, \ldots, X_{v, N_{r}-1}^{r}\right]^{\mathrm{T}} \quad$ where $0 \leq v \leq V-1$, that is to say, $\boldsymbol{X}^{r}=\sum_{v=0}^{V-1} \boldsymbol{X}_{v}^{r}$. Notice that there are only $N_{r} / V$ non-zero values at the corresponding position of $\boldsymbol{X}_{v}^{r}$ while the other positions are zeros. A partial transmission sequence is defined as:

$x_{v}^{r}=\operatorname{IFFT}\left\{X_{v}^{r}\right\}=\frac{1}{\sqrt{N_{r}}} \sum_{k=0}^{N_{r}-1} X_{v}^{r} \mathrm{e}^{j \frac{2 \pi k n}{N_{r}}}$

Without loss of generality, we just consider the first sub-band, i.e., $r=1$, and define $N_{1}=N$. To reduce the probability of peak signals, each partial transmission sequence is multiplied with the phase rotating vector $b_{v}^{u}(0 \leq v \leq V-1,0 \leq u \leq U-1)$, which is generated from Hadamard matrix with the order of $V$ and $U$ is the number of alternative phase rotating vectors. Under different phase rotating vectors, various time-domain sequences representing the same data symbol are obtained as $x^{u}=\sum_{v=0}^{V-1} b_{v}^{u} x_{v}$. Hence, the optimal phase factor can be calculated as:

$b^{\tilde{u}}=\arg \min _{b^{u} \in B^{V}}\left(\max _{0 \leq n \leq N-1}\left|\sum_{v=0}^{V-1} b_{v}^{u} x_{v, n}\right|\right)$

Then, by combining $b^{\tilde{u}}$ with pilot sequence $\mathrm{P}=\left[p_{0}, p_{1}, \ldots, p_{N-1}\right]$, the optimized pilot sequence can be obtained as $P^{\tilde{u}}=b^{\tilde{u}} \cdot \mathrm{P}$. These phase rotating vector and the pilot signal will be used for symbol recovery at the receiver later. Afterwards are the $\mathrm{CP}$ padding and sub-band filtering. Different from the OFDM, the filtering here is for suppressing the out-of-band leakage. Particularly, when it comes to fading channel, there is a slight difference that no phase optimization is needed for the data symbol and pilot symbol in the $1^{\text {st }}$ slot of the 1 st transmission time interval (TTI).

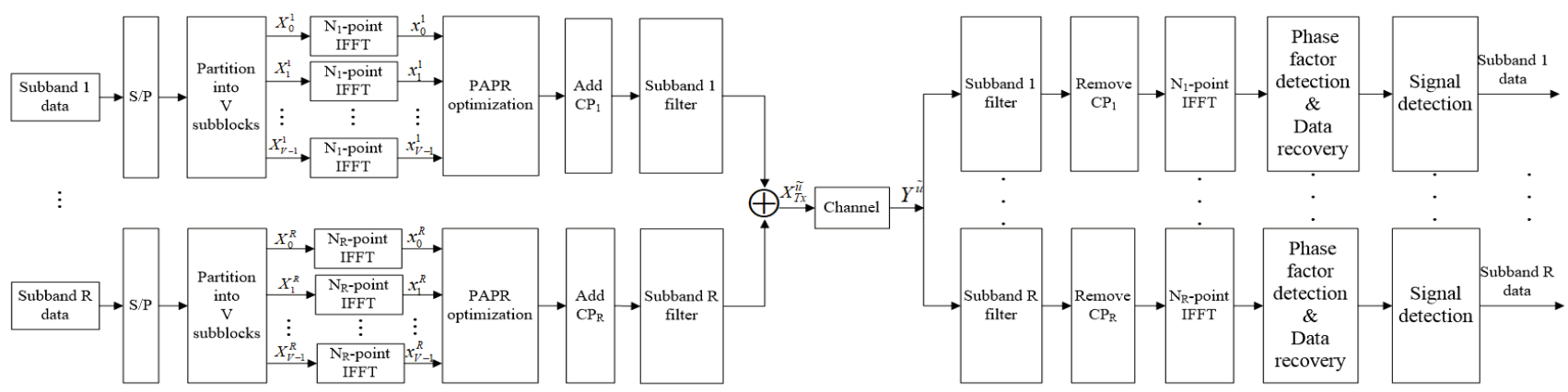

(a) Transmitter

Figure 2 Block diagram of f-OFDM transceiver with PTS: (a) transmitter (b) receiver

\subsection{Detector without SI}

The detector is designed to find out the phase factor $b^{\tilde{u}}$ carried by pilot then to recover symbols without SI transmission. At the receiver, after sub-band matchedfiltering, CP-removing, and the $N$-point FFT, the received signal can be expressed as:

$Y^{\tilde{u}}(k)=H(k) \cdot X^{\tilde{u}}(k)+N(k)$ where $\quad Y^{\tilde{u}}(k)=F F T\left(y^{\tilde{u}}\right)=\frac{1}{\sqrt{N}} \sum_{N=0}^{N_{1}-1} y^{\tilde{u}}(n) \mathrm{e}^{-j \frac{2 \pi k n}{N}}$, $k=0,1, \ldots, N-1, H(k)$ is the channel frequency response and $N(k)$ is AWGN. Then $Y^{\tilde{u}}$ is partitioned into $V$ disjoint subsequences $Y_{v}^{\tilde{u}}$, similar to the operation at the transmitter. This series of operations is also performed on pilot symbols and get the sequence $[Q(n), n=0,1, \ldots, N-1]$. 


\subsubsection{AWGN Channel}

The frequency response $H(k)$ of the AWGN channel is a unit matrix. The cross-correlation of the received pilot sequence and the original pilot sequence carrying the phase factor is computed as:

$$
R_{p q}(m)=\left[P(n) \cdot b^{u}(n)\right] \cdot Q(-n)
$$

where $b^{u}(n) \in \boldsymbol{B}, n=0,1, \ldots, N-1$. The detected optimal phase factor is obtained by

$$
b_{v}^{\tilde{u}}=\underset{b^{u}(n) \in B}{\operatorname{argmax}} R_{p q}(m)=\underset{b^{u}(n) \in B}{\operatorname{argmax}}\left[p(n) \cdot b^{u}(n)\right] * Q(-n)
$$
by

The pilot symbols and data symbols can be recovered

$$
\begin{aligned}
& Y_{v}=\sum_{v=0}^{V-1} Y_{v}^{\tilde{u}} / b_{v}^{\tilde{u}} \\
& Q^{\prime}(n)=Q(n) / b^{\tilde{u}}(n)
\end{aligned}
$$

where $n=0, \ldots, N-1$.

\subsubsection{Fading Channel}

Different from the AWGN channel, the frequency response of a fading channel is varying during signal transmission. However, this variation between adjacent TTIs can be assumed to be negligible because the time interval between pilot symbols in adjacent TTIs is smaller than the coherent time of the system. Therefore, we can update the channel frequency response per TTI.

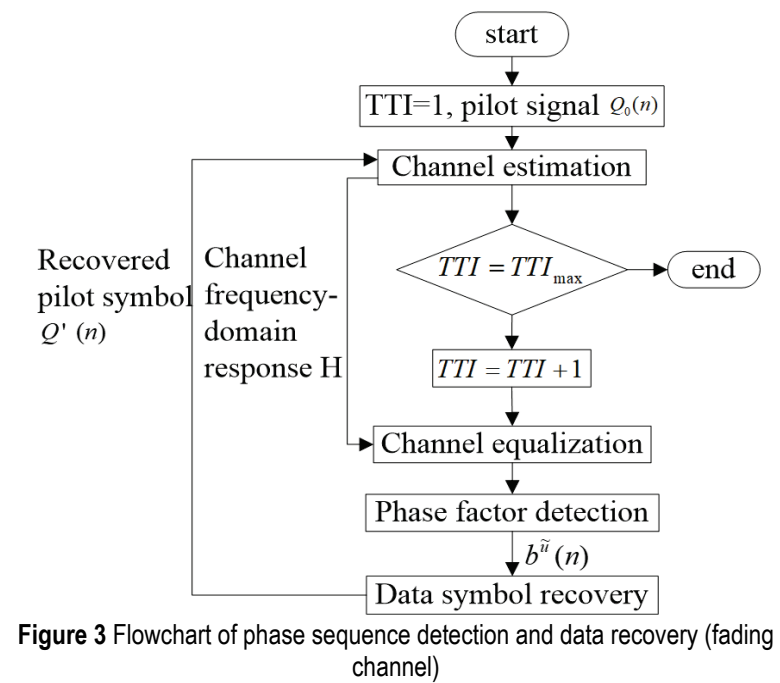

The procedure of phase sequence detection and data recovery is illustrated in Fig. 3 and Algorithm 1. The channel frequency response $H_{n-1}$ in the $(n-1)^{\text {th }}$ TTI is derived through channel estimation and then cached to the next TTI. After updating the channel frequency response, we derive the equalized pilot signal $[\hat{Q}(n), n=0,1, \ldots, N-1]$ using the current received pilot symbol $[Q(n), n=0,1, \ldots, N-1]$. Then phase factor detection is carried out with the same procedure as that in AWGN channel.

$$
R_{p q}(m)=\left[P(n) \cdot b^{u}(n)\right] \cdot \hat{Q}(-n)
$$

and

$$
b_{v}^{\tilde{u}}=\underset{b^{u}(n) \in B}{\operatorname{argmax}} R_{p q}(m)=\underset{b^{u}(n) \in B}{\operatorname{argmax}}\left[p(n) \cdot b^{u}(n)\right] \cdot \hat{Q}(-n) .
$$

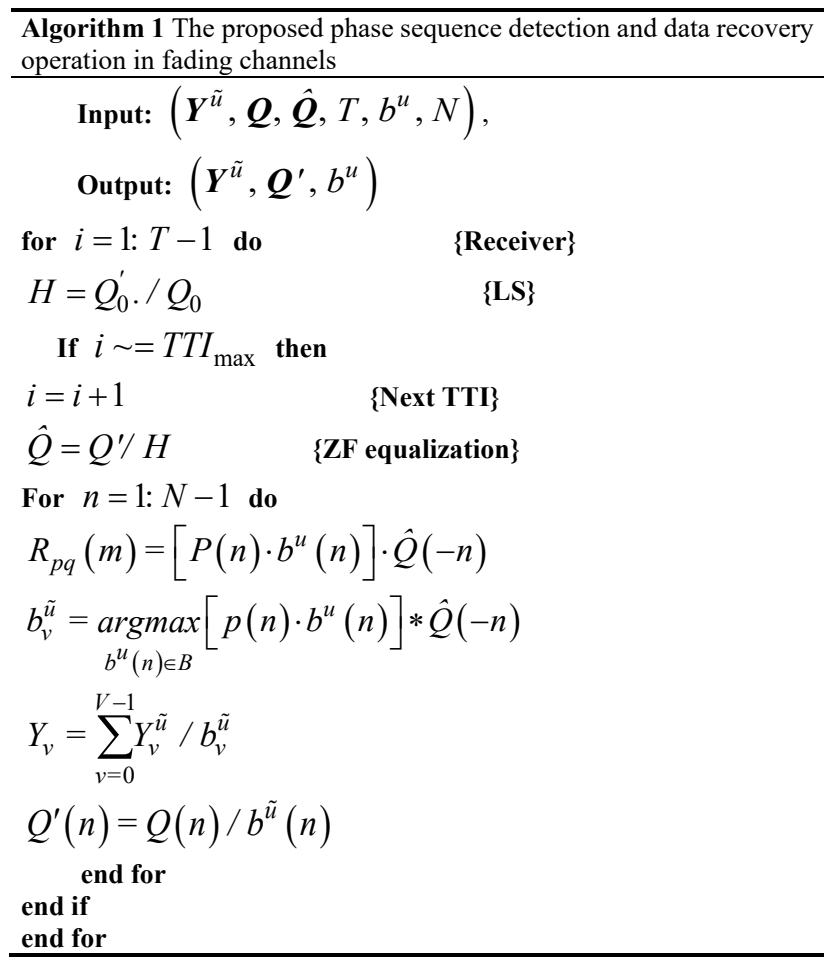

Then channel estimation is operated again using the phase recovered data and pilot symbols. The resulting frequency response $H_{n}$ is cached in a cyclic procedure. In particular, the phase optimization sequence of the data symbol and pilot symbol in the $1^{\text {st }}$ slot of the $1^{\text {st }}$ TTI is a unit sequence to guarantee the accuracy of channel estimation.

\section{SIMULATION RESULTS}

In this section, the performance of the proposed PTS scheme is compared with other conventional PAPR reduction schemes. The parameters and the time-frequency resource structure used in this simulation are shown in Tab. 1 and Fig. 4, respectively. The number of sub-bands and other parameters of the f-OFDM system can be implemented flexibly according to different scenarios and the proposed PTS scheme is carrier out in each sub-band independently. Thus the parameters of different sub-bands except the number of subsequences will not have an impact on the PAPR reduction and detection. In this simulation, we use 48 subcarriers per symbol and a pilot symbol is inserted every six data symbols in a slot. Each symbol is partitioned into eight subsequences. 


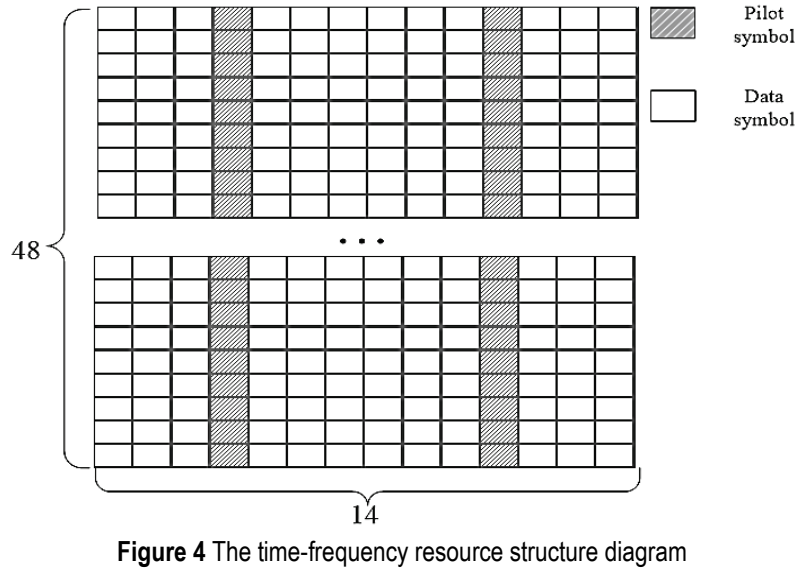

Table 1 Simulation parameters

\begin{tabular}{|l|l|}
\hline \multicolumn{1}{|c|}{ Parameters } & \multicolumn{2}{c|}{ Sub-band 1 } \\
\hline Duplex & FDD \\
\hline Antenna config. & SISO \\
\hline Sampling rate & $30.72 \mathrm{Mb} / \mathrm{s}$ \\
\hline Subcarrier spacing & $15 \mathrm{kHz}$ \\
\hline Subcarrier/symbol & 48 \\
\hline FFT size & 2048 \\
\hline Symbol duration & $1 / 15 \mathrm{k}=66.67 \mu \mathrm{s}$ \\
\hline TTI/ms & 1 \\
\hline Symbol/TTI & 14 \\
\hline \multirow{2}{*}{ CP length } & 160 samples for symbol 1 \\
\cline { 2 - 3 } & 144 samples for symbol 2:6 \\
\hline CRC & 24 bit \\
\hline Modulation & QPSK \\
\hline Turbo coding rate & $1 / 3$ \\
\hline Subsequence & 8 \\
\hline
\end{tabular}

Fig. 5 shows the OOBE performance of conventional OFDM, f-OFDM without PAPR reduction schemes and fOFDM with clipping, clipping and filter (CAF) and the proposed PTS schemes. Compared to the conventional OFDM, the OOBE can be suppressed effectively due to the shaping filter in the f-OFDM system. Moreover, the proposed PTS scheme preserves the strength of low-OOBE of original f-OFDM without introducing extra performance loss while the signal distortion schemes will result in severe OOBE.

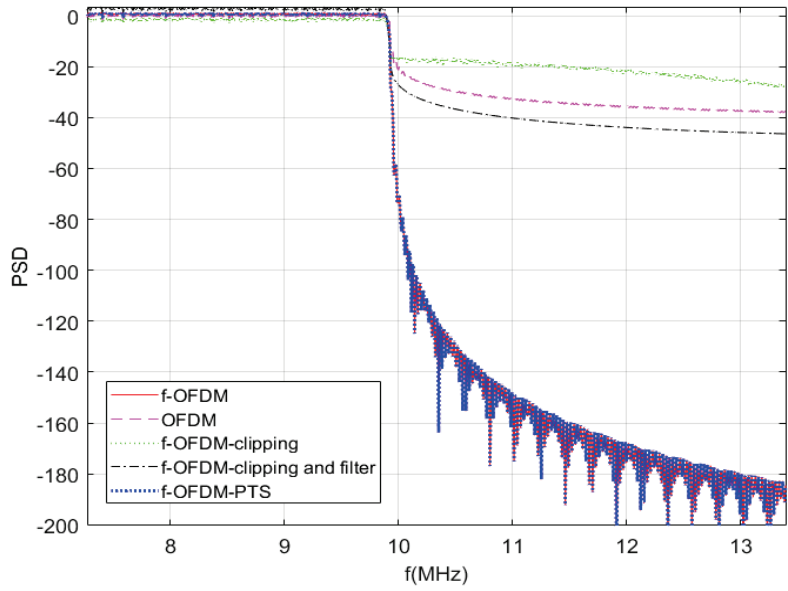

Figure 5 OOBE performance of proposed PTS scheme and conventional PAPR reduction scheme (clipping, clipping and filter)

Fig. 6 compares the PAPR reduction performance of original-PTS, proposed PTS and f-OFDM without PAPR reduction. The proposed PTS exhibits the same PAPR reduction performance as the original PTS scheme in the
AWGN channel, which means intensive PAPR suppression in the f-OFDM system. While in the fading channel, the PAPR reduction performance is slightly worse than that in the AWGN channel because the phase optimization sequence of the data symbol and pilot symbol in the $1^{\text {st }}$ slot of the $1^{\text {st }}$ TTI is a unit sequence to guarantee the accuracy of channel estimation. Under the same PAPR reduction condition, the proposed PTS scheme can save 11\% bandwidth utilization compared to the traditional PTS scheme with the transmission of SI and the performance gain will be improved with the increase of the number of subsequences.

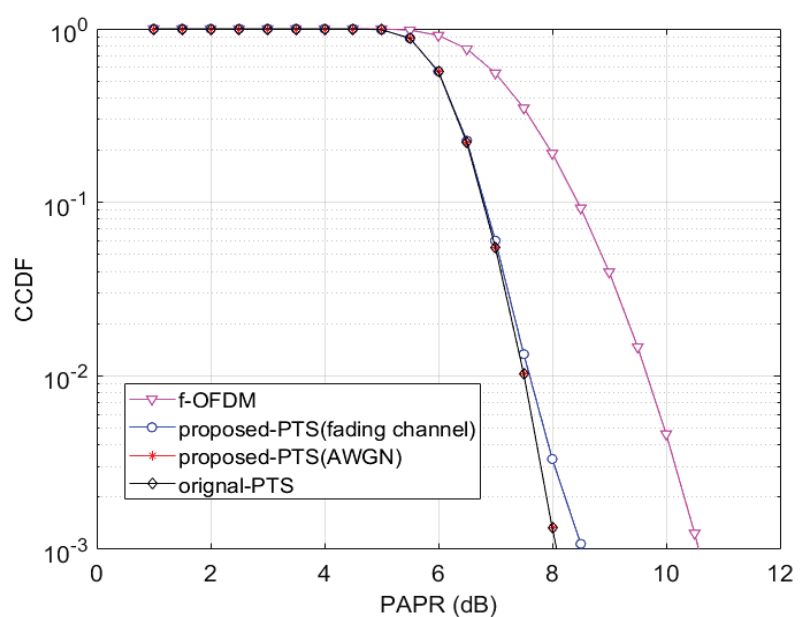

Figure 6 PAPR reduction performance of OFDM, f-OFDM, original-PTS and proposed PTS scheme

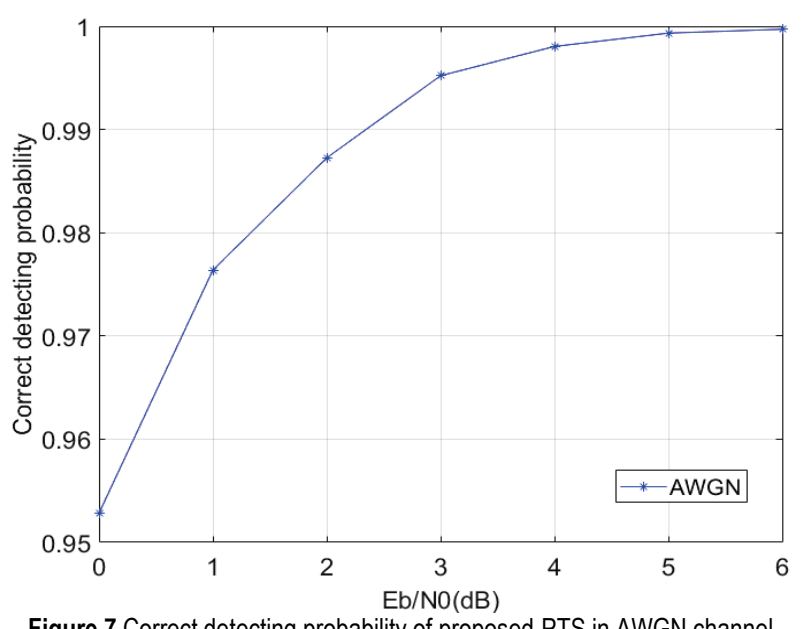

Figure 7 Correct detecting probability of proposed-PTS in AWGN channel

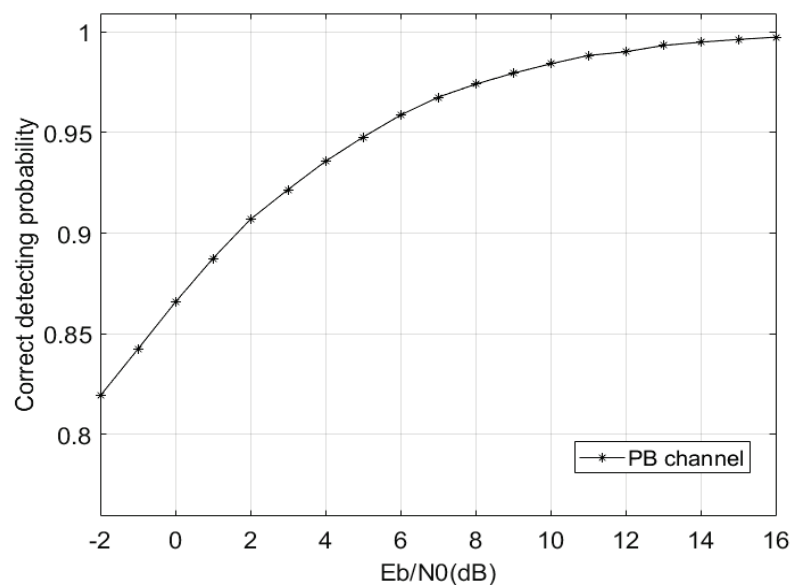

Figure 8 Correct detecting probability of proposed-PTS in PB channel 
The correct detecting probability of the proposed PTS scheme in AWGN channel and PB fading channel is depicted in Fig. 7 and Fig. 8, respectively. It is obvious that the correct detecting probability of the conventional PTS method with transmitting known SI is $100 \%$. Therefore, the performance of the proposed PTS scheme is almost equal to the conventional PTS method when the correct detecting probability approaches 1 while the transmission of the SI is no longer needed. The results reveal that a high correct detecting probability could be achieved in AWGN channel. The probability of error detection is close to $1 \%$ at $E_{b} / N_{0}=$ $3 \mathrm{~dB}$, which can be ignored. Despite the performance loss in PB channel, the correct detection probability can reach $95 \%$ at $E_{b} / N_{0}=6 \mathrm{~dB}$. Moreover, compared to other PTS scheme without SI transmission, the proposed scheme provides comparable correct probability without the use of complicated detector (like ML detector).

\section{CONCLUSION}

A new and simple PTS scheme without SI transmission has been proposed for PAPR reduction of $\mathrm{f}$ OFDM systems. The correlation property of the Hadamard sequence and the transparency of the pilot signals have been utilized to detect the optimal phase factor and recover the data and pilot symbols, while a new iterative detection is adopted for a fading channel scenario. Without sacrificing the PAPR reduction performance, the proposed scheme has a compact channel overhead and higher channel utilization than the conventional method which needs SI transmission. Moreover, it is capable of high correct detecting probability and a lower complexity without using the high-complexity detector. In summary, the proposed PTS scheme could solve the high-PAPR problem of f-OFDM simply and effectively, and make fOFDM more applicable for future generation communications.

\section{REFERENCES}

[1] Fettweis, G., Krondorf, M., \& Bittner, S. (2009).GFDM generalized frequency division multiplexing. In Vehicular Technology Conference, 2009, IEEE, 1-4. https://doi.org/10.1109/VETECS.2009.5073571

[2] Vakilian, V., Wild, T., Schaich, F., Brink, S. T., \& Frigon, J. F. (2014). Universal-filtered multi-carrier technique for wireless systems beyond LTE. In GLOBECOM Workshops, 223-228. https://doi.org/10.1109/GLOCOMW.2013.6824990

[3] Abdoli, J., Jia, M., \& Ma, J. (2015). Filtered OFDM: A new waveform for future wireless systems. In IEEE International Workshop on Signal Processing Advances in Wireless Communications, 66-70. https://doi.org/10.1109/SPAWC.2015.7227001

[4] Banelli, P., Buzzi, S., Colavolpe, G., \& Modenini, A. (2014). Modulation formats and waveforms for 5G networks: Who will be the heir of OFDM?: An overview of alternative modulation schemes for improved spectral efficiency. IEEE Signal Processing Magazine, 31(6), 80-93. https://doi.org/10.1109/MSP.2014.2337391

[5] Goel, A., Poddar, P., \& Agrawal, M. (2018). A novel quadrilateral companding transform for PAPR reduction in OFDM systems. Digital Signal Processing, 85, 113-123. https://doi.org/10.1016/j.dsp.2018.11.002

[6] Kang, S. G., Kim, J. G., \& Joo, E. K. (1999). A novel subblock partition scheme for partial transmit sequence
OFDM. IEEE Transactions on Broadcasting, 45(3), 333-338. https://doi.org/10.1109/11.796276

[7] Bae, K., Shin, C., \& Powers, E. J. (2013). Performance analysis of ofdm systems with selected mapping in the presence of nonlinearity. IEEE Transactions on Wireless Communications, 12(5), 2314-2322. https://doi.org/10.1109/TWC.2013.040213.120968

[8] Meng Z., Gong Y., Zhu X., Tu X., Xie J. (2019) LowPAPR Precoder Design for Large-Scale MIMO-OFDM Systems. In: Patnaik S., Jain V. (eds) Recent Developments in Intelligent Computing, Communication and Devices. Advances in Intelligent Systems and Computing, vol 752. Springer, Singapore. https://doi.org/10.1007/978-981-10-8944-2_92

[9] Renuka, N. \& Satya, S. M. (2019). Peak-to-Average Power Ratio Performance of Transform Precoding-Based Orthogonal Frequency Division Multiplexing Offset Quadrature Amplitude Modulation. Smart Intelligent Computing and Applications, 105, 241-248. https://doi.org/10.1007/978-981-13-1927-3_25

[10] Yang, L., Soo, K. K., Li, S. Q., \& Siu, Y. M. (2011). PAPR reduction using low complexity PTS to construct of OFDM signals without side information. IEEE Transactions on Broadcasting, 57(2), 284-290. https://doi.org/10.1109/TBC.2011.2122870

[11] Guan, L., Jiang, T., Qu, D., and Zhou, Y. (2010). Joint channel estimation and PTS to reduce peakto- averagepower radio in OFDM systems without side information. IEEE Signal Processing Letters, 17(10), 883-886. https://doi.org/10.1109/LSP.2010.2066562

[12] Tan, G., Li, Z., Su, J., \& Zhang, H. (2013). Superimposed training for PTS-PAPR reduction in OFDM: A side information free data recovery scheme. In International ICST Conference on Communications and NETWORKING in China, 694-698. https://doi.org/10.1109/ChinaCom.2013.6694682

[13] Kalaiselvan, E., Elavarasan, P., \& Nagarajan, G. (2013). PAPR reduction of OFDM signals using pseudo random PTS without side information. In 2013 International Conference on Communication and Signal Processing, 29-33. https://doi.org/10.1109/iccsp.2013.6577008

[14] Joo, H. S., Kim, K. H., No, J. S., \& Shin, D. J. (2017). New PTS schemes for PAPR reduction of OFDM signals without side information. IEEE Transactions on Broadcasting, $P P(99), 1-9$.

[15] Mabrouk, M. B., Chafii, M., Louet, Y., \& Bader, F. (2017). A precoding-based PAPR reduction technique for UFOFDM and Filtered-OFDM modulations in $5 \mathrm{G}$ systems. In European Wireless 2017, 23 ${ }^{\text {th }}$ European Wireless Conference.

[16] Cho, Y. J., Kim, K. H., Woo, J. Y., Lee, K. S., No, J. S., \& Shin, D. J. (2017). Low-complexity PTS schemes using dominant time-domain samples in OFDM systems. IEEE Transactions on Broadcasting, PP(99), 1-6. https://doi.org/10.1109/TBC.2017.2662228

\section{Contact information}

\section{Yaqiu PENG}

Shanghai Advanced Research Institute, Chinese Academy of Sciences No. 99 Haike Road, Zhangjiang Hi-Tech Park, Pudong, Shanghai 201210, China University of Chinese Academy of Sciences, Beijing, 100049, China E-mail: pengyq_sari@163.com

\section{Mingqi LI}

(Corresponding author)

Shanghai Advanced Research Institute, Chinese Academy of Sciences,

No. 99 Haike Road, Zhangjiang Hi-Tech Park, Pudong, Shanghai 201210, China 